Document downloaded from:

http://hdl.handle.net/10251/153038

This paper must be cited as:

Bueso Rodenas, E.; Serrano Salom, R.; Pallas, V.; Sanchez Navarro, JA. (2017). Seed tolerance to deterioration in arabidopsis is affected by virus infection. Plant Physiology and Biochemistry. 116:1-8. https://doi.org/10.1016/j.plaphy.2017.04.020

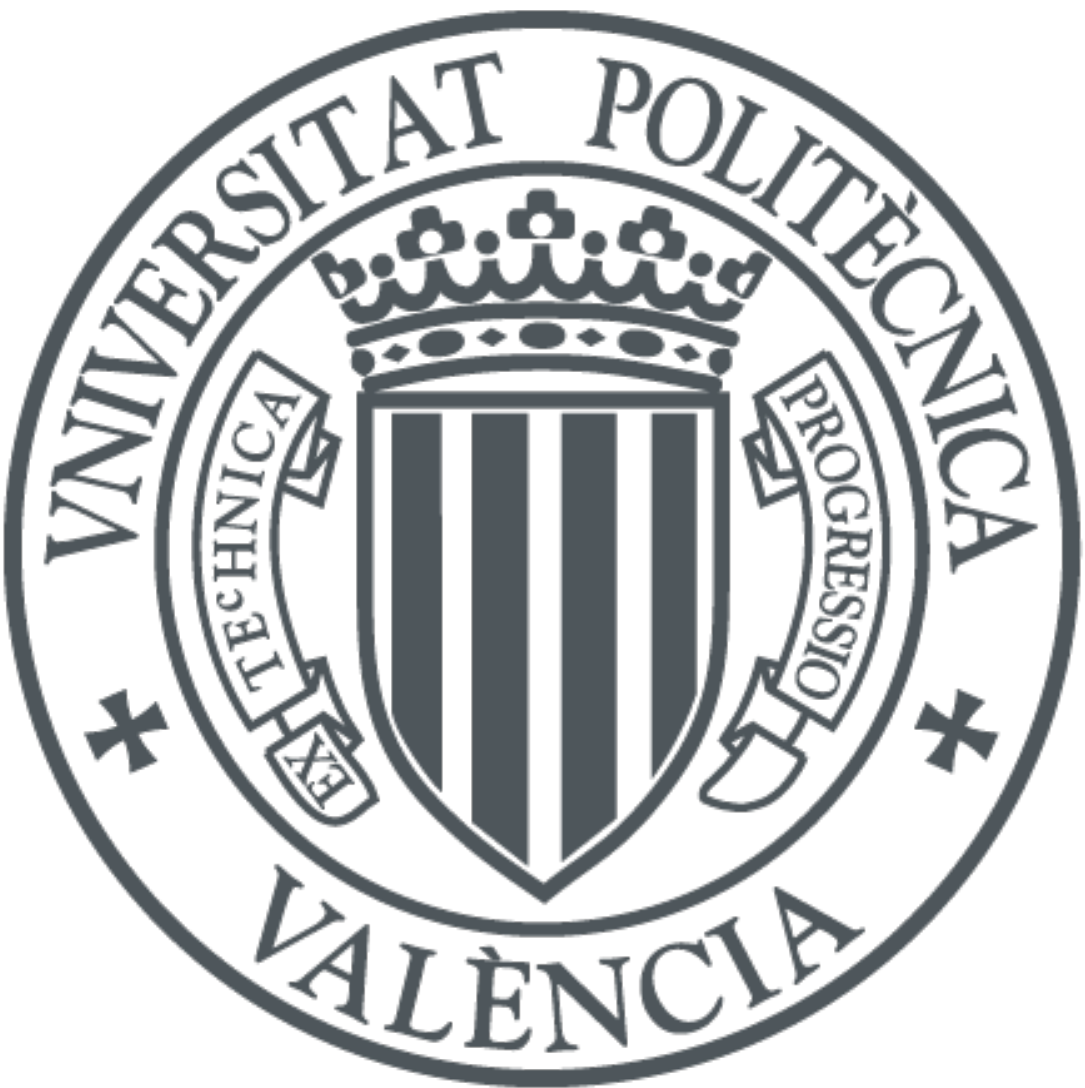

The final publication is available at

https://doi.org/10.1016/j.plaphy.2017.04.020

Copyright Elsevier

Additional Information 


\section{SEED DETERIORATION IN ARABIDOPSIS IS AFFECTED BY VIRUS INFECTION}

Eduardo Bueso, Ramón Serrano, Vicente Pallás, and Jesús A. Sánchez-Navarro*

Instituto de Biología Molecular y Celular de Plantas (IBMCP), Consejo Superior de investigaciones Científicas-Universidad Politécnica de Valencia, Valencia, Spain.

Eduardo Bueso: edubueso@gmail.com

Ramón Serrano: rserrano@ibmcp.upv.es

Vicente Pallás: vpallas@ibmcp.upv.es

Jesús A. Sánchez-Navarro*: jesanche@ibmcp.upv.es.

*Author for correspondence: Jesús Ángel Sánchez-Navarro

Instituto de Biología Molecular y Celular de Plantas (CSIC-UPV)

Ingeniero Fausto Elio s/n, 46022 Valencia, Spain

Tel: 34963877989 ; Fax: 34963877859

E-mail: jesanche@ibmcp.upv.es

Running title: Virus infection stress in seed survival 


\begin{abstract}
Seed longevity is the period during which the plant seed is able to germinate. This property is strongly influenced by environment conditions experienced by seeds during their formation and storage. In the present study we have analysed how the biotic stress derived from the infection of Cauliflower mosaic virus (CaMV), Turnip mosaic virus(TuMV), Cucumber mosaic virus (CMV) and Alfalfa mosaic virus (AMV) affects seed tolerance to deterioration measuring germination rates after an accelerated aging treatment. Arabidopsis wild type plants infected with AMV and CMV rendered seeds with improved tolerance to deterioration when compared to the non-inoculated plants. On the other hand, CaMV infection generated seeds more sensitive to deterioration. No seeds were obtained from TuMV infected plants. Similar pattern of viral effects was observed in the double mutant athb22 athb25, which is more sensitive to accelerated seed aging than wild type. However, we observed a significant reduction of the seed germination for CMV (65\% vs 55\%) and healthy (50\% vs 30\%) plants in these mutants. The seed quality differences were overcomed using the $A$. thaliana athb25-1D dominant mutant, which over accumulated gibberellic acid (GA), except for TuMV which generated some siliques with low seed tolerance to deterioration. For AMV and TuMV (in athb25-1D), the seed quality correlated with the accumulation of the messengers of the gibberellin 3-oxidase family, the mucilage of the seed and the GA1. For CMV and CaMV it was not a good correlation suggesting that other factors are affecting seed viability.
\end{abstract}

Keywords: Arabidopsis thaliana; Gibberellins; Plant hormones; Seed deterioration; Virus infection. 


\section{INTRODUCTION}

Seed longevity, defined as the period over which seed remains viable, is a crucial property of plants that will affect the maintenance of plant genetic diversity. Many factors have been described to influence seeds longevity and the rate of aging is strongly influenced by environment (moisture content, temperature and initial seed quality) and genetic factors (Sano et al. 2016).

The existing knowledge of molecular mechanisms involved in seed longevity is limited and the few genes studied belong to three different groups. The first one includes seed developmental mutants. The alteration of key regulators of seed maturation (e.g. leafy cotyledon1,_lec1 or abscisic acid insensitive3, abi3) exhibit significantly reduced seed viability upon storage (Sugliani et al. 2009). Next mutant groups are those related to alteration of the seed coat or testa. This natural barrier protects the embryo and seed reserves from biotic and abiotic stresses (Rajjou and Debeaujon 2008). The third mutant group corresponds to protection and repair systems that prevent seed vigour loss (Sattler et al. 2004) or maintain genome integrity in plants (Waterworth et al. 2010).

Accumulation of defense proteins and specific enzymes associated to protein repair are also related to seed longevity. High accumulation of small heat shock proteins improved tolerance to aging (Prieto-Dapena et al. 2006) while the protein repair enzyme L-isoaspartyl methyltransferase (PIMT) plays a key role in the long-term survival of seeds (Oge et al. 2008). Enzymes playing roles in the detoxification of reactive oxygen species (e.g. glutathione peroxidase and glutathione reductase) and toxic cyanide compounds (e.g. $\beta$-mercaptopyruvate sulfurtransferase) are important to extend seed longevity (Rajjou and Debeaujon 2008).

Hormones are also associated to seed longevity through their role in the differentiation of the seed coat and in the maturation phase (Braybrook and Harada 2008, Bueso et al. 2014). The increased level of the stress hormone abscisic acid (ABA) during the maturation phase of seed development prepares the seed to resist desiccation and aging in a dormant state. By the other hand, arabidopsis ABA response mutants are deficient in accumulation of seed proteins protecting against desiccation (Braybrook and Harada 2008). The growth hormone gibberellin (GA) has been recently associated to seed deterioration (Bueso et al. 2014). Seeds from wild-type plants treated with GA and from a quintuple DELLA mutant (with constitutive GA signalling) are more 
tolerant to seed vigour. Also, the overexpression of COG1/DOF1.5 (Park et al. 2003), encoding a DOF ("DNA-binding with One Finger") transcription factor that attenuates light responses mediated by phytochromes, increases GA in the mother plant and reduces the permeability of the seed coat by increasing suberin accumulation in the palisade layer (Bueso et al. 2016).

After being challenged by biotic or abiotic stresses, plants initiate several responses that are modulated by different plant hormones, which their signalling pathways are internally interconnected to generate an efficient stress response (Navarro et al. 2008, Bari and Jones 2009, Nishiyama et al. 2013, Verma et al. 2016). The involvement of plant hormones in seed deterioration permits to connect this trait with plan responses to abiotic and biotic stresses (Verma et al. 2016). The main hormones mediating plant defense response to pathogens and abiotic stresses are ABA, salicylic acid (SA), jasmonic acid (JA) and ethylene (ET) (Bari and Jones 2009, Nakashima and Yamaguchi-Shinozaki 2013), in which ABA is responsible for plant defence against abiotic stresses while SA, JA and ET are the main components against biotic stress (Bari and Jones 2009).

SA is synthetized by plants in response to a wide range of pathogens, including plant viruses after recognition of viral effectors by resistance (R)-gene products that trigger incompatible interactions. Such interactions generated several responses to limit viral propagation at the infection site, including accumulation of reactive oxygen species (ROS) and pathogenesis-related (PR) proteins, induction of the hypersensitive response (HR), callose deposition and programmed cell death (PCD) (Baebler et al. 2014). JA is involved in resistance to necrotrophic pathogens and insect infestation (Thaler et al. 2004) and ET does not appear to be essential for plant resistance against viruses but can be involved in symptom development (Geri et al. 2004). The mechanisms of stress-response are not restricted to these hormones and recent studies provided substantial evidences for the crosstalk of ABA, SA, JA and ET with auxins (IAA), GAs and cytokinins (CKs) in regulating plant defense response (Navarro et al. 2008, Bari and Jones 2009, Nishiyama et al. 2013).

To accomplish their life cycle, plant viruses must hijack the functions of different host factors altering, in some instances, the plant physiology and hormonal homeostasis (Pallas and Garcia, 2011; Collum and Culver, 2016). It has been shown that virus infection has a major effect on resource allocation to growth and reproduction, infection resulting in a general reduction of resources allocated to both 
traits (Pagan et al., 2008) and that some viruses directly affect the viability of seeds (Sastry, 2013). However, how virus infection affects seed deterioration has not been studied yet. In the present study we have analyzed how biotic stress derived from the infection of different plant viruses affect seed quality. We have selected four different viruses differing in genome organization, the host range or the mode of transmission. The Cauliflower mosaic caulimovirus (CaMV) has a double-stranded DNA genome whereas the Turnip mosaic potyvirus (TuMV), Cucumber mosaic cucumovirus (CMV) and Alfalfa mosaic alfamovirus (AMV) are single strand RNA (ssRNA) viruses with 1 (TuMV) or 3 (CMV and AMV) RNAs, respectively. In addition, CMV and AMV are seed transmitted whereas CaMV and TuMV are transmitted through aphids. Seeds obtained from infected wild type Arabidopsis thaliana Columbia plants and two derived mutants that present improved (athb25-1D) or reduced (athb22 athb25) seed tolerance to deterioration (Bueso et al. 2014) revealed different results according to the virus used. In addition, we show the impact of virus infection on the hormonal balance.

\section{MATERIAL AND METHODS}

\section{Plant inoculations.}

To produce TuMV, CMV, AMV and CaMV infectious extracts, adequate host plants for each virus were inoculated with an initial infectious material. Thus, to produce the initial TuMV infection, Nicotiana benthamiana plants were mechanically inoculated with a plasmid containing a TuMV cDNA (GenBank accession number AF530055.2) corresponding to isolate YC5 from calla lily (Zante-deschia sp.) under the control of CaMV 35S promoter (Chen et al. 2007). A mix of the three 5'-capped transcripts corresponding to the CMV-Fny or AMV-425 strains were produced in vitro and mechanically inoculated in $N$. benthamiana plants. In the case of the AMV, the inoculum contained also few micrograms of purified AMV coat protein. The infection with CaMV was started by mechanical inoculation of Plasmid pCaMVW260 (Scholelz and Shepherd 1988) in Brassica rapa L. 'Just Right'.At $\approx 14$ days postinoculation (dpi), when infection symptoms were clearly observable, symptomatic tissues were harvested, aliquoted, frozen and stored at $-80^{\circ} \mathrm{C}$. Aliquots of the different infected tissues were then ground with a mortar and pestle in the presence of liquid $\mathrm{N}_{2}$ and homogenized in 34 volumes of inoculation buffer $(50 \mathrm{mM}$ potassium phosphate, $\mathrm{pH} 8.0)$. The resulting crude extracts were used to mechanically inoculate batches of 3-week-old A. thatiana 
ecotype Col-0 wild type (wt) and athb25-1D or athb22 athb25 mutants. The A. thaliana athb25-1D line overexpress the homeobox 25 transcription factor (ATHB25) and presents improved seed tolerance to deterioration under both natural and accelerated aging conditions meanwhile the mutant athb22-25 has loss function of both ATHB25 and the most similar zinc finger homeodomain $A T H B 22$, that exhibits reduced tolerance to accelerated aging (Bueso et al. 2014).

\section{Dot blot assays.}

Dot blot analysis was performed using total RNA extracted from inoculated leaves at $7 \mathrm{dpi}$, as described previously (Sanchez-Navarro et al. 1997). Alternatively, the plants were also analyzed using a fast citrate buffer protocol that does not require any special manipulation (Sanchez-Navarro et al. 1996). The samples were directly applied onto nylon membranes positively charged (Roche Diagnostics GmbH, Manheim, Germany), air-dried and cross-linked by UV crosslinker $(700 \times 100 \mu \mathrm{J} / \mathrm{cm} 2)$. Hybridization and detection was conducted as previously described (Sanchez-Navarro et al. 1996) using a dig-riboprobe (Roche Mannheim, Germany) complementary to part of the coat protein gene of the corresponding virus. Chemiluminiscent detection using CSPD reagent as substrate was performed as recommended by the manufacturer (Roche Diagnostics GmbH, Manheim, Germany). Films were exposed for 30 minutes.

\section{Plant Material and Growth Conditions}

Arabidopsis (Arabidopsis thaliana) plants were grown and germinated on Murashige and Skoog (MS) plates with sucrose as described (Bueso et al. 2014). Plants treated with GA3 were sprayed twice per week with a $50 \mathrm{mM}$ solution. We used the following Arabidopsis strains: wild-type Columbia, activation-tagging mutant overexpressing ATHB25 (athb25-1D), double loss of function of ATHB25 (NASC line N665321; athb25-2) and ATHB22 (NASC line N667869).

\section{Accelerated Aging assays}

This procedure was a modification of the "basal thermotolerance assay" (Tejedor-Cano et al. 2010). Seeds were imbibed in water and stratified for 3 days at $4^{\circ} \mathrm{C}$, and incubated at $42^{\circ} \mathrm{C}$ for 1 to 2 days (relative humidity of $100 \%$ ). 


\section{Quantification of hormones}

Plant material, corresponding to a mix of inoculated and systemic leaves at 8 dpi, was suspended in $80 \%$ methanol-1\% acetic acid containing internal standards and mixed by shaking during one hour at $4{ }^{\circ} \mathrm{C}$. The extract was kept a $-20{ }^{\circ} \mathrm{C}$ overnight and then centrifuged and the supernatant dried in a vacuum evaporator. The dry residue was dissolved in $1 \%$ acetic acid and passed through an Oasis HLB (reverse phase) column as described in Seo et al. (2011). The dried eluate was dissolved in 5\% acetonitrile-1\% acetic acid, and the hormones were separated using an autosampler and reverse phase UPHL chromatography (2.6 $\mu \mathrm{m}$ Accucore RP-MS column, $50 \mathrm{~mm}$ length x $2.1 \mathrm{~mm}$ i.d.; ThermoFisher Scientific) with a 5 to $50 \%$ acetonitrile gradient containing $0.05 \%$ acetic acid, at $400 \mu \mathrm{L} / \mathrm{min}$ over $14 \mathrm{~min}$. The hormones were analyzed with a Q-Exactive mass spectrometer (Orbitrap detector; ThermoFisher Scientific) by targeted Selected Ion Monitoring (SIM). The concentrations of hormones in the extracts were determined using embedded calibration curves and the Xcalibur 2.2 SP1 build 48 and TraceFinder programs. The internal standards for quantification of each of the different plant hormones were the deuterium-labelled hormones (Plant Hormones Quantification Service at the Institute for Plant Molecular and Cell Biology, http://www.ibmcp.csic.es/index_en.php\#)

\section{Staining of the Mucilage with Ruthenium Red}

Seeds were imbibed in water for 3 min before the application of $0.2 \%(\mathrm{w} / \mathrm{v})$ aqueous ruthenium red solution for $15 \mathrm{~min}$. Photographs were taken with Nikon's Eclipse E600 research microscope, and mucilage area was measured with the Javabased image-processing program ImageJ.

\section{Molecular Techniques}

Total RNA was extracted from 28-d-old (1 week after infection) wild type plants using NucleoSpin RNA II kit (Macherey-Nagel, Düren, Germany) and $3 \mu \mathrm{g}$ RNA was reverse transcribed using the Maxima first-strand cDNA synthesis Kit for RT-qPCR (Thermo Fisher Scientific, Waltham, MA, USA) according to the manufacturer's instructions. PCR amplifications were performed on first cDNA strand corresponding to $150 \mathrm{ng}$ of total RNA and each reaction was performed in triplicate in a total volume of 
$25 \mu 1$. Quantitative (real-time) PCR (qRT-PCR) was performed using an Applied Biosystems 7500 Real-Time PCR System (Thermo Fisher Scientific) with the 5x PyroTaq EvaGreen qPCR Mix Plus (ROX) (Cultek S.L.U., Madrid, Spain) according to the manufacturer's protocol. Data are the mean of three biological samples. PCR amplification specificity was confirmed with a heat-dissociation curve (from 60 to 95 ${ }^{\circ} \mathrm{C}$ ). Efficiency of the PCR was calculated and different internal standards (PP2AA3 or At5g55840) were selected depending on the efficiency of the primers (Czechowski et al. 2005). Relative mRNA abundance was calculated using the comparative DCt method (Pfaffl 2001). The primers utilized were for GA3OX1 5' -gatctcctcttctcegctgct-3' and 5'gagggatgttttcaccggtg-3, for GA3OX2 5'-ctgccgetcatcgacctc-3'and 5'agcatggcccacaagagtg-3, for GA3OX3 5'-gatcacaccaagtactgcggtataa-3 and $5^{\prime}$ ttccatttcgtccacgtattctt-3, for GA3OX4 5'-cgetacactcttatggeccg-3' and 5'tccatcacattgcagaactcg-3.

\section{RESULTS}

\section{Seed tolerance to deterioration in Arabidopsis is affected by virus infection}

To characterize how virus infection affects seed longevity, we collected seeds from A. thaliana plants wild type (wt) and two mutants presenting improved (athb251D) or reduced (athb22 athb25) seed longevity under both natural and accelerated aging conditions (Bueso et al. 2014), infected with the corresponding TuMV, CMV, AMV and CaMV virus. Previously, we confirmed by dot blot analysis that all plants were infected by the corresponding virus (data not shown). We observed that virus infection affected Arabidopsis development in different ways (Fig. 1A). While AMV practically did not affect growth, CMV and CaMV significantly inhibited it, and TuMV was able to stop totally the growth in wild type and in mutant athb22 athb25. However, the athb25$1 D$ line infected with TuMV was able to generate some siliques.

Next, we evaluated seed tolerance to deterioration by performing an accelerated aging assay (Fig. 1B). In the case of seeds derived from the athb25-1D line, the virus infection practically did not affect germination after accelerated aging treatment, with a percentage of germination about $75 \%$ However, TuMV was able to reduce germination after accelerated aging by more than $40 \%$, indicating the drastic effect of such biotic stress in seed quality. 
On the other hand, the effect of virus infection in seeds derived from wt and double mutant athb22 athb25 plants was similar. In the case of TuMV most of the infected plants failed to develop a flower stalk and no seed production was obtained. Surprisingly, AMV and CMV infection led the plant to produce seeds with improved tolerance to deterioration meanwhile CaMV infection generated seeds with a low percentage of germination after accelerated aging. When both wild type and athb22 athb25 plants were compared we observed the same percentage of germination in the accelerated aging assay for seeds derived from AMV (75\%) and CaMV (25\%) infected plants meanwhile a significant reduction of the seed germination for CMV $(65 \% \mathrm{vs}$ $55 \%$ ) and healthy (50\% vs 30\%) plants was observed. Apparently, AMV and CaMV overcome the loss of function of both ATHB25 and ATHB22 transcription factors.

The most likely mechanism by which the overexpression of ATHB25 produces seeds resistant to aging is based in the regulation of GA, key hormones in the formation of seed coat, specially of the outer integument where mucilage pectin is produced. According to this, there is a direct relationship between ATHB25 and this polysaccharide (Bueso et al. 2014). Accordingly, we studied mucilage formation after virus infection in the three lines (Fig. 1C). As expected, gain of function of ATHB25 produced more mucilage than wild type, meanwhile the production of this compound was significantly reduced in the case of the double mutant athb22 athb25. In the case of AMV, the overproduction of mucilage in the wild type reaches similar levels than in athb25-1D, although in the case of the double mutant the increase of the mucilage was very limited. On the other hand, CMV and CaMV did not affect the production of the mucilage in wild type and athb22 athb255, although a reduction of this compound was evident in the activation tagging mutant. Finally, the production of mucilage in the athb25-1D mutant was totally impaired after TuMV infection.

\section{Virus infection regulates GA3 oxidases}

The importance of the contribution of gibberellins to the formation of arabidopsis seed coat is already known (Kim et al. 2005). In order to test whether mucilage changes after virus infection are due to changes in gibberellin content during seed formation, we carry out a transcriptomic study of gibberellin 3-oxidase (GA3OX), an enzyme family that catalyses the final step in the synthesis of bioactive gibberellins (Olszewski et al. 2002). We observed different patterns according to the accumulation 
of the four messenger RNAs analysed. Two viruses (AMV and CaMV) induced the accumulation of two messengers RNAs and the reduction of only one RNA meanwhile CMV and TuMV induced the reduction of two or the four gibberellin 3-oxidase messengers, respectively (Fig. 2).

In the case of AMV and CaMV infections, both viruses induced GA3OX2 but differently. Thus, meanwhile AMV increased fivefold the messenger RNA, CaMV was only 1.2 times. The second induced gene differed between both viruses being GA3OX3 (1.6 times) for AMV or $G A 3 O X 1$ (1.2 times) for CaMV. The weak induction of these oxidases and the significant repression of GA3OX4 could explain the poor germination showed by seeds coming from plants infected with CaMV. On the other hand the great increased accumulation of the gibberellin 3-oxidase messengers, specially the GA3OX2 (5 times), in the AMV infected plants, correlates with the mucilage formation and the improved seed germination (25\%) after accelerating aging. Finally, the absence of induced genes in the CMV infected plants do not correlate with seed germination data in the accelerating aging assays, indicating that other factors different from seed testa modification are contributing to seed quality.

\section{Hormonal alterations after AMV and TuMV infections}

Next, we decided to carry out an hormone content study in the A. thaliana wt after AMV and TuMV infection, two viruses showing opposite effects in the different performed analysis (Fig. 3). The hormones analyzed were gibberellin (GA1), abscisic acid (ABA), jasmonic acid (JA), salicylic acid (SA) and auxin (IAA), all described with some relationship with plant pathology. The results revealed two different behaviors. The TuMV infected plants accumulated sevenfold less bioactive gibberellin GA1 (GA4 was undetectable in all the analyzed tissues) than the non-inoculated plants. In addition, the content in two stress-related hormones were increased two (ABA) or 10 (SA) folds, indicating a strong plant response to the virus infection. On the other hand, AMV was able to control the typical SA overproduction after pathogen infection. In addition, this virus strongly reduced the level of JA and the accumulation of GA1 was appreciably higher comparing with uninfected plants. The rest of the analyzed hormones were not significantly affected. From the point of view of hormones concentration, the AMVinfected plants have an antagonistic effect to that observed for the TuMV-infected plants. 


\section{TuMV impaired-growth is recovered by exogenous GAs application}

During the analysis, we observed that TuMV was able to arrest the growth of the infected plants with no siliques production in the wild type genotype. These strong phenotypic changes were partly overcomed by infecting the GAs over-producer line athb25-1D, indicating that GAs play a critical role in the TuMV biotic stress. In the next step, we were interested to check if the GAs positive effect could be also observed by external application of the plant hormone. To do this, A. thaliana Col-0 wt plants were treated exogenously by GAs (50 $\mu \mathrm{M}$ GA3) at $-7,-2,0$ and +4 days, being 0 the TuMV infection day (Fig. 4). At 8 days post inoculation $(+8)$ we observed that the treated plants were able to significantly increase the total fresh weight, with values close to the healthy plants, allowing silique production although did not reverse the inhibition of stalk development (Fig. 4A and 4C). We also observed that the GA treatment only affected virus symptomatology but not prevented or limited virus infection (Fig. 4B).

\section{DISCUSSION}

To accomplish their life cycle, plant viruses interact and/or interfere with host components that, in turn, in some instances can alter, among other cellular processes, the hormonal homeostasis (Pallas and Garcia, 2011; Collum and Culver, 2016). By the other hand, different hormones such as ABA and GAs have been recently demonstrated to play a key role in the seed longevity (Braybrook and Harada 2008, Bueso et al. 2014). Thus, it is reasonable to think that viruses, by altering hormonal signalling pathways could have a significant effect on seed longevity. Although it has been described for long time that virus infection affects seed germination, the effects of these pathogens on seed tolerance to deterioration have never been studied. In the present analysis, we have evaluated how four different plant viruses, differing in their genomic organization and mode of transmission, affected seed quality. We observed different phenotypic patterns after virus infection, ranging from unaffected plants (AMV), followed by the slight (CMV), strong (CaMV) or totally (TuMV) growth reduction. This strong effect on the failure on developing a flower stack in TuMV-infected plants has previously observed for the UK 1 isolate (Sanchez et al., 2015). Interestingly, the phenotypic changes correlated with seed quality, pointing to the idea that such 
differences could be due to hormonal miss-regulation. The main hormone related to these characters is GA. For instance, mutants of positive regulators of GA signalling exhibit dwarfed phenotype with compact leaves, delayed flowering, reduced fertility and poor germination (Davies 2013). This idea is also supported by the observation that seed quality differences were overcome using the A. thaliana athb25-1D line which over accumulated GAs (Bueso et al. 2014), except for the TuMV virus. In this case it was possible to obtain at least some siliques with seeds showing low tolerance to deterioration. On the other hand, seeds obtained from the AMV and CMV infected plants showed improved seed tolerance to deterioration when compared to the healthy plants. Interestingly, and unlike CaMV and TuMV, both viruses are transmitted by seed (Sastry 2013). It is tempting to speculate that seed-transmitted viruses could favour a long period of seed fertility to ensure them to be transmitted to the plant progeny. Remarkably, this result is consistent with the life-history theory that states that interaction with low virulent parasites will result in a delay in host reproduction, which allows for compensation of parasite damage (Gandon et al., 2002).

GAs have recently been demonstrated to play a key role in seed vigour by reducing the permeability of the seed coat due to the increase of suberin accumulation in the palisade layer of the seed coat (Bueso et al. 2014; 2016). Our results revealed that the seed quality observed for AMV and TuMV was correlated with the accumulation of the RNA messengers of the gibberellin 3-oxidase protein family and with mucilage accumulation. For CMV and CaMV it was not a clear correlation suggesting that other factors are affecting seed vigour. Also, in the case of CMV it has been described that the virus reduced the accumulation of active GAs in cucumber plants (Ben-Tal and Marco 1980) which was correlated with the slight plant growth reduction of the Arabidopsis CMV-infected plants.

In the case of the AMV, the improved seed germination (25\%) observed in the accelerating aging assays correlated with the increase of fivefold of the expression of GA3OX2 and a high GA1 accumulation. It is interesting to note that the same messenger was incremented only 2.5 -fold in the A. thaliana athb25-1D line that over-accumulated GAs (Bueso et al. 2014). The observation that GA and ABA have an antagonistic relationship, with their pathways integrated by the DELLA proteins (Golldack et al. 2013), could point to the idea that AMV may block the plant defence mechanism by increasing GAs accumulation. In this sense, we observe that the ABA levels were comparable to the healthy plant. We observed that infection with AMV decreased JA 
but left unaffected ABA, SA and IAA. The lack of effect of decreased JA on SA is not contrast with the report that plants impaired in the JA pathway failed to accumulte SA (Zhu et al. 2014). We would expect that AMV, by decreasing JA, should decrease SA but this is not observed.

Recently, it has been shown that the coat protein of AMV promotes the accumulation of SA and JA via the interaction with the transcription factor ILR3 (Aparicio and Pallás 2016). However, the accumulation of the JA in Arabidopsis lossof-function ILR3 mutant (ilr3.2) after AMV infection was increased more than 10-fold, with low virus accumulation. These results suggest that AMV could be controlling JA accumulation via the CP-ILR3 interaction. Altogether these results support the idea that modulation of hormone signalling pathway by plant viral proteins constitute another mechanism to counteract host defences (Collum and Culver, 2016), especially relevant in the case of AMV since no typical counter defence proteins (e.g. silencing suppressor, etc) has been described, so far. In any case, further analyses are required to check whether the different phenotypes, observed after virus infection, are or not the consequence of the viral mechanism to counteract host defences.

In the case of TuMV, we observed a dramatic phenotypic alteration that could be the consequence of the plant defence response or the incapacity of the virus to modulate hormone signalling pathways. Interesting, we observed that the main plant stress hormones are up-regulated (ABA, JA and SA) even when some of them are antagonistic (e.g. JA vs SA or ABA vs SA). However, simultaneous up-regulation of ABA and SA or JA and SA has been described in plant defence responses (Alazem et al. 2013). Also, the observation that GAs are down regulated by ABA and JA, via the DELLA proteins (Golldack et al. 2013, Heinrich et al. 2013) supports the low GAs accumulation observed in the TuMV infected plants, probably by interfering to the GAs synthesis since part of the phenotype could be rescue by external GAs application. In this scenario, Sanchez et al. (2015) only observed a very slight increment in size and growth rate in response to GA3. The fact that in our case plants recovered more significantly could be due to the differences in the treatment made. Indeed, our plants were treated before and after virus inoculation whereas in the work by Sanchez et al. (2015) plants were only treated after inoculation.

In summary, presented data show that the biotic stress derived from plant virus infection affects seed longevity and that effect is closely related to GAs accumulation. 
Also, we show that this effect is virus-dependent and probably related to the differential accumulation of plant hormones.

\section{Author contributions}

All authors listed, have made substantial, direct and intellectual contribution to the work. The authors have no conflict of interest to declare.

\section{Acknowledgements}

We thank L. Corachán and I. Martínez for their excellent technical assistance. This work was supported by grant BIO2014-54862-R from the Spanish Direccion General de Investigacion Cientifica y Tecnica (DGICYT) and the Prometeo Program GV2014/010 from the Generalitat Valenciana.

Fig. 1. Seed tolerance to deterioration in Arabidopsis is affected by virus infection. (A) Different virus effects in the development of the plant from athb25-1D, wild type (WT) and double mutant athb22 athb25. Inside of each panel, from left to right: mock (healthy plants without treatment), Alfalfa mosaic virus (AMV), Cauliflower mosaic virus (CaMV), Cucumber mosaic virus (CMV) and Turnip mosaic virus (TuMV). (B) Seeds obtained at 30 dpi from infected and not infected lines athb25-1D, wild type (WT) and double mutant athb22 athb25 were subjected to accelerated aging for $24 \mathrm{~h}$ and sown on MS plates. The percentage of germination was recorded at the indicated days (X-axis). The results are averages of six experiments with 100 seeds per line, and error bars indicate the SE. Untreated lines germinated more than $99 \%$ after 3 days. (C) Mucilage staining of representative seeds from infected and not infected lines athb25$1 D$, wild type (WT) and double mutant athb22 athb25. (D) Measure of the surrounded mucilage area. Each value is the mean of 20 seeds chosen at random from two different batches, 10 each. Error bars indicate SE.

Fig. 2. Virus infection regulates GA3 oxidases expression. Quantitative RT-PCR analyses were performed in triplicate on RNA samples from 28-d-old (1 week after infection) wild type plants. Relative values refer to the expression levels of the GA3 oxidases with respect to mock, which was taken as 1. Error bars indicate SE. 
Fig. 3. AMV and TuMV produce hormonal changes in Arabidopsis. Endogenous GA1, ABA, JA, SA and IAA levels from 28-d-old (1 week after infection) wild type plants. Means were calculated from four independents experiments and error bars indicate SE.

Fig. 4. Impaired plant growth produced by TuMV is recovered by GAs. (A) Representative growth of 28-d-old wild type Arabidopsis plants after 1 week TuMV infection with or without $50 \mu \mathrm{M} \mathrm{GA}_{3}$ exogenous treatment. (B) TuMV infection detection by dot blot hybridization. Total RNA extracted from healthy and infected plants were applied on nylon membranes and hybridized using a dig-RNA probe complementary to the part of the TuMV coat protein gene. The films were exposed for 30 minutes. (C) Quantification of the fresh weight (FW) aerial growth of 28-d-old Arabidopsis plants after 1-week TuMV infection with or without $50 \mu \mathrm{M} \mathrm{GA}_{3}$ treatment. Mock correspond to healthy plants without treatment. Means were calculated from four independents experiments and error bars indicate SE.

\section{LITERATURE CITED}

Alazem, M., Lin, K.Y., Lin, N.S., 2013. The Abscisic Acid Pathway Has Multifaceted Effects on the Accumulation of Bamboo mosaic virus. Mol. Plant-Microbe Interact. 27, 177-189.

Aparicio, F., Pallás, V., 2016. The coat protein of Alfalfa mosaic virus interacts and interferes with the transcriptional activity of the bHLH transcription factor ILR3 promoting salicylic-dependent defence signalling response. Mol. Plant Pathol. doi:10.1111/mpp.12388.

Baebler, Š., Witek, K., Petek, M., Stare, K., Tušek-Žnidarič, M., Pompe-Novak, M., Renaut, J., Szajko, K., Strzelczyk-Żyta, D., Marczewski, W., Morgiewicz, K., Gruden, K., Hennig, J., 2014. Salicylic acid is an indispensable component of the Ny-1 resistance-gene-mediated response against Potato virus $\mathrm{Y}$ infection in potato. J. Exp. Bot. 65, 1095-1109.

Bari, R., Jones, J.D.G., 2009. Role of plant hormones in plant defence responses. Plant Mol. Biol. 69, 473-488.

Ben-Tal, Y., Marco, S., 1980. Qualitative changes in cucumber gibberellins following cucumber mosaic virus infection. Physiol. Plant Pathol. 16, 327-336.

Braybrook, S.A., Harada, J.J., 2008. LECs go crazy in embryo development. Trends Plant Sci. 13, 624-630.

Bueso, E., Munoz-Bertomeu, J., Campos, F., Brunaud, V., Martinez, L., Sayas, E., Ballester, P., Yenush, L., Serrano, R., 2014. ARABIDOPSIS THALIANA HOMEOBOX25 uncovers a role for Gibberellins in seed longevity. Plant Physiol. 164, 999-1010.

Bueso, E., Munoz-Bertomeu, J., Campos, F., Martinez, C., Tello, C., MartinezAlmonacid, I., Ballester, P., Simon-Moya, M., Brunaud, V., Yenush, L., Ferrandiz, C., 
Serrano, R., 2016. Arabidopsis COGWHEEL1 links light perception and gibberellins with seed tolerance to deterioration. Plant J. 87(6), 583-596.

Chen, C.-C., Chen, T.-C., Raja, J.A.J., Chang, C.-A., Chen, L.-W., Lin, S.-S., Yeh, S.D., 2007. Effectiveness and stability of heterologous proteins expressed in plants by Turnip mosaic virus vector at five different insertion sites. Virus Res. 130, 210-227.

Chen, C.C., Chao, C.H., Chen, C.C., Yeh, S.D., Tsai, H.T., Chang, C.A., 2003. Identification of Turnip mosaic virus isolates causing yellow stripe and spot on calla lily. Plant Dis. 87, 901-905.

Collum, T.D., Culver, J.N., 2016. The impact of phytohormones on virus infection and disease. Curr. Opin. Virol. 17, 25-31.

Czechowski, T., Stitt, M., Altmann, T., Udvardi, M.K., Scheible, W.R., 2005. Genomewide identification and testing of superior reference genes for transcript normalization in Arabidopsis. Plant Physiol. 139, 5-17.

Davies, P., 2013. Plant Hormones: Physiology, Biochemistry and Molecular Biology. Springer Netherlands.

Gandon S., Agnew P., Michalakis Y., 2002. Coevolution between parasite virulence and host life-history traits. Am. Nat. 160, 374-388.

Geri, C., Love, A.J., Cecchini, E., Barrett, S.J., Laird, J., Covey, S.N., Milner, J.J., 2004. Arabidopsis mutants that suppress the phenotype induced by transgene-mediated expression of Cauliflower mosaic virus (CaMV) gene VI are less susceptible to CaMVinfection and show reduced ethylene sensitivity. Plant Mol. Biol. 56, 111-124.

Golldack, D., Li, C., Mohan, H., Probst, N., 2013. Gibberellins and abscisic acid signal crosstalk: living and developing under unfavorable conditions. Plant Cell Rep. 32, 1007-1016.

Heinrich, M., Hettenhausen, C., Lange, T., Wunsche, H., Fang, J., Baldwin, I.T., Wu, J., 2013. High levels of jasmonic acid antagonize the biosynthesis of gibberellins and inhibit the growth of Nicotiana attenuata stems. Plant J. 73, 591-606.

Kim, Y.C., Nakajima, M., Nakayama, A., Yamaguchi, I., 2005. Contribution of gibberellins to the formation of Arabidopsis seed coat through starch degradation. Plant Cell Physiol. 46, 1317-1325.

Nakashima, K., Yamaguchi-Shinozaki, K., 2013. ABA signaling in stress-response and seed development. Plant Cell Rep. 32, 959-970.

Navarro, L., Bari, R., Achard, P., Lisón, P., Nemri, A., Harberd, N.P., Jones, J.D.G., 2008. DELLAs control plant immune responses by modulating the balance of jasmonic acid and salicylic acid signaling. Curr. Biol. 18(9), 650-655.

Nishiyama, R., Watanabe, Y., Leyva-Gonzalez, M.A., Ha, C.V., Fujita, Y., Tanaka, M., Seki, M., Yamaguchi-Shinozaki, K., Shinozaki, K., Herrera-Estrella, L., Tran, L.S., 2013. Arabidopsis AHP2, AHP3, and AHP5 histidine phosphotransfer proteins function as redundant negative regulators of drought stress response. Proc. Natl. Acad. Sci. U. S. A. $110,4840-4845$.

Oge, L., Bourdais, G., Bove, J., Collet, B., Godin, B., Granier, F., Boutin, J.P., Job, D., Jullien, M., Grappin, P., 2008. Protein repair L-isoaspartyl methyltransferase 1 is involved in both seed longevity and germination vigor in Arabidopsis. Plant Cell 20, 3022-3037.

Olszewski, N., Sun, T.P., Gubler, F., 2002. Gibberellin signaling: biosynthesis, catabolism, and response pathways. Plant Cell 14 Suppl, S61-80.

Pagan, I., Alonso-Blanco, C., Garcia-Arenal, F., 2008. Host responses in life-history traits and tolerance to virus infection in Arabidopsis thaliana. PLoS pathog. 4.

Pallas, V., Garcia, J.A., 2011. How do plant viruses induce disease? Interactions and interference with host components. J. Gen. Virol. 92, 2691-2705. 
Park, D.H., Lim, P.O., Kim, J.S., Cho, D.S., Hong, S.H., Nam, H.G., 2003. The Arabidopsis COG1 gene encodes a Dof domain transcription factor and negatively regulates phytochrome signaling. Plant J. 34, 161-171.

Pfaffl, M.W., 2001. A new mathematical model for relative quantification in real-time RT-PCR. Nucleic Acids Res. 29, e45.

Prieto-Dapena, P., Castaño, R., Almoguera, C., Jordano, J., 2006. Improved resistance to controlled deterioration in transgenic seeds. Plant Physiol. 142, 1102-1112.

Rajjou, L., Debeaujon, I., 2008. Seed longevity: survival and maintenance of high germination ability of dry seeds. C. R. Biol. 331, 796-805.

Sanchez, F., Manrique, P., Mansilla, C., Lunello, P., Wang, X., Rodrigo, G., LopezGonzalez, S., Jenner, C., Gonzalez-Melendi, P., Elena, S.F., Walsh, J., Ponz, F., 2015. Viral strain-specific differential alterations in arabidopsis developmental patterns. Mol. Plant Microbe Interact. 28, 1304-1315.

Sanchez-Navarro, J.A., Cano, E.A., Pallas, V., 1996. Non-radioactive molecular hybridization detection of Carnation mottle virus in infected carnations and its comparison to serological and biological techniques. Plant Pathol. 45, 375-382.

Sanchez-Navarro, J.A., Reusken, C.B.E.M., Bol, J.F., Pallas, V., 1997. Replication of alfalfa mosaic virus RNA 3 with movement and coat protein genes replaced by corresponding genes of Prunus necrotic ringspot ilarvirus. J. Gen. Virol. 78, 3171-3176.

Sano, N., Rajjou, L., North, H.M., Debeaujon, I., Marion-Poll, A., Seo, M., 2016. Staying alive: molecular aspects of seed longevity. Plant Cell Physiol. 57, 660-674.

Sastry, K.S., 2013. Seed-borne plant virus diseases. Springer India.

Sattler, S.E., Gilliland, L.U., Magallanes-Lundback, M., Pollard, M., DellaPenna, D., 2004. Vitamin e is essential for seed longevity and for preventing lipid peroxidation during germination. Plant Cell 16, 1419-1432.

Scholelz, J.E., Shepherd, R.J., 1988. Host range control of Cauliflower mosaic virus. Virology 162, 30-37.

Seo, M., Jikumaru, Y., Kamiya, Y., 2011. Profiling of hormones and related metabolites in seed dormancy and germination studies. Methods Mol. Biol. 773, 99-111.

Sugliani, M., Rajjou, L., Clerkx, E.J., Koornneef, M., Soppe, W.J., 2009. Natural modifiers of seed longevity in the Arabidopsis mutants abscisic acid insensitive3-5 (abi3-5) and leafy cotyledon1-3 (lec1-3). New Phytol. 184, 898-908.

Tejedor-Cano, J., Prieto-Dapena, P., Almoguera, C., Carranco, R., Hiratsu, K., OhmeTakagi, M., Jordano, J., 2010. Loss of function of the HSFA9 seed longevity program. Plant Cell Environ. 33, 1408-1417.

Thaler, J.S., Owen, B., Higgins, V.J., 2004. The role of the jasmonate response in plant susceptibility to diverse pathogens with a range of lifestyles. Plant Physiol. 135, 530538.

Verma, V., Ravindran, P., Kumar, P.P., 2016. Plant hormone-mediated regulation of stress responses. BMC Plant Biol. 16, 86.

Waterworth, W.M., Masnavi, G., Bhardwaj, R.M., Jiang, Q., Bray, C.M., West, C.E., 2010. A plant DNA ligase is an important determinant of seed longevity. Plant J. 63, 848-860.

Zhu, F., Xi, D.H., Yuan, S., Xu, F., Zhang, D.W., Lin, H.H., 2014. Salicylic acid and jasmonic acid are essential for systemic resistance against tobacco mosaic virus in Nicotiana benthamiana. Mol. Plant Microbe Interact. 27, 567-577. 